\title{
Comparison of Minimally Invasive Surgery and Abdominal Surgery Among Patients With Cervical Cancer
}

\author{
DOMINIK RATIU*, CHRISTIAN LUNCESCU*, BERND MORGENSTERN, CHRISTIAN EICHLER, \\ BERTHOLD GRÜTTNER, SEBASTIAN LUDWIG, PETER MALLMANN and FABINSHY THANGARAJAH \\ Department of Obstetrics and Gynecology, University Hospital Cologne and Medical Faculty, Cologne, Germany
}

\begin{abstract}
Background/Aim: The aim of this study was to evaluate the difference in clinical outcomes in patients with histologically confirmed cervical cancer of the uterus treated with either laparoscopy or laparotomy with curative intent between 2011 and 2017 at the Department of Gynecology and Obstetrics of University Hospital Cologne. Materials and Methods: This retrospective analysis included all patients who received surgical treatment with curative intent between January 2011 and December 2017 for stages IAI to IIB cervical carcinoma. Patients receiving primary or secondary surgery after neoadjuvant chemotherapy were also included. Results: In total, 75 patients were included, of whom 34 patients underwent minimally invasive surgery and 41 underwent open surgery. Neoadjuvant chemotherapy was performed in 10 patients in the minimally-invasive group and in 14 patients in the laparotomy group. Statistically, no significant difference in overall survival (OS) was observed in both groups. Disease-free survival showed a significant difference in favor of the minimally invasive group. Conclusion: Minimally invasive surgical therapy for cervical cancer improves disease-free-survival. Prospective trials are needed to further confirm these results.
\end{abstract}

In developed countries, cervical cancer is responsible for $15 \%$ of cancers among women $(1,2)$. In total, $80 \%$ of these cancers arise from squamous cell dysplasia, whereas $15 \%$ are adenocarcinomas, and 5\% are clear cell adenocarcinomas (1, $3)$. Knowledge of the molecular mechanisms underlying the development and metastases of cervical cancer, except for human papilloma virus infection, is still limited $(1,4)$.

*These Authors contributed equally to this study.

Correspondence to: Dr. med. Fabinshy Thangarajah, University Hospital Cologne, Department of Obstetrics and Gynecology, Medical Faculty, Kerpener Str. 34, 50931 Cologne, Germany. Tel: +49 22147886545, e-mail: Fabinshy.Thangarajah@uk-koeln.de

Key Words: Cervical cancer, neoadjuvant therapy, OS, DFS, surgery.
Smoking and multiparity are known epidemiological cofactors for the development of cervical cancer $(1,5)$.

Therapy modalities depend on the patients' clinical stage. Therapeutical options vary from primary surgery or neoadjuvant chemotherapy followed by surgery or definitive radiochemotherapy to palliative systemic therapy (6).

New advances led to an improvement of the overall survival (OS) and disease-free survival in patients with metastatic and advanced cervical cancer (7).

The previously published Laparoscopic Approach to Cervical Cancer (LACC) study (8) showed that minimally invasive radical hysterectomy is associated with lower rates of disease-free survival and OS than open abdominal radical hysterectomy among women with early-stage cervical cancer. This led to a change in practice in many institutions. Hence, the aim of this study was to evaluate the difference in clinical outcomes in patients with histologically-confirmed cervical cancer of the uterus treated with either laparoscopy or laparotomy with curative intent at the Department of Gynecology and Obstetrics of University Hospital Cologne.

\section{Materials and Methods}

In this retrospective hospital-based analysis, patients with histologically confirmed curable cervical cancer treated between 2011 and 2017 in the University Hospital Cologne were included (Table I).

Inclusion criteria. Patients with histologically proven stages IA1 to IIB cervical carcinoma without distant metastasis who received surgical treatment with curative intent.

Exclusion criteria. Patients who did not receive any guideline-based therapy due to noncompliance or patients for whom lymphadenectomy was not indicated according to their tumor stage (FIGO IA1 without risk factors).

A retrospective analysis was performed for the following parameters: patient age, FIGO tumor stage, histomorphological tumor type, lymphatic and venous infiltration, and grading at first presentation. The tumor size was documented as the longest diameter either post surgery on the basis of histopathological findings or pre-therapeutically before neoadjuvant chemotherapy on the basis of sonography or MRI imaging. 
Table I. Patient characteristics.

\begin{tabular}{|c|c|c|c|}
\hline Characteristics & Minimally invasive surgery $(n=33)$ & Open surgery $(n=41)$ & $p$-Value \\
\hline Age (years) & $41.16 \pm 9.15$ & $46.39 \pm 13.37$ & 0.057 \\
\hline Histologic subtype n (\%) & & & 0.487 \\
\hline Squamous-cell carcinoma & $24(70.6 \%)$ & $26(63.4 \%)$ & \\
\hline Adenocarcinoma & $9(26.5 \%)$ & $11(26.8 \%)$ & \\
\hline Adenosquamous carcinoma & $1(2.9 \%)$ & $4(9.8 \%)$ & \\
\hline Stage of disease $\mathrm{n}(\%)$ & & & 0.134 \\
\hline FIGO IA1 & $9(26.5 \%)$ & $1(2.4 \%)$ & \\
\hline FIGO IA2 & $3(8.8 \%)$ & $1(2.4 \%)$ & \\
\hline FIGO IB1 & $9(26.5 \%)$ & $21(51.2 \%)$ & \\
\hline FIGO IB2 & $3(8.8 \%)$ & $6(14.6 \%)$ & \\
\hline FIGO IIA1 & $1(2.9 \%)$ & $2(4.9 \%)$ & \\
\hline FIGO IIA2 & $2(5.9 \%)$ & $4(9.8 \%)$ & \\
\hline FIGO IIB & $7(20.6 \%)$ & $6(14.6 \%)$ & \\
\hline Grade $(\%)$ & & & 0.035 \\
\hline G1 & $2(6.3 \%)$ & $0(0 \%)$ & \\
\hline G2 & $20(62.5 \%)$ & $19(46.3 \%)$ & \\
\hline G3 & $10(31.3 \%)$ & $22(53.7 \%)$ & \\
\hline Tumor size $(\mathrm{cm})$ & $2.63 \pm 2.19$ & $3.26 \pm 1.64$ & 0.221 \\
\hline LVSI & & & 0.010 \\
\hline $\mathrm{L} 1$ & $5(16.7 \%)$ & $18(47.4 \%)$ & \\
\hline L0 & $25(83.3 \%)$ & $20(52.6 \%)$ & \\
\hline VSI & & & 0.394 \\
\hline V1 & $4(13.3 \%)$ & $2(5.3 \%)$ & \\
\hline V0 & $26(86.7 \%)$ & $36(94.7 \%)$ & \\
\hline Recurrence & & & 0.030 \\
\hline Yes & $2(5.9 \%)$ & $10(24.4 \%)$ & \\
\hline No & $32(94.1 \%)$ & $31(75.6 \%)$ & \\
\hline Death & & & 0.222 \\
\hline Yes & $2(5.9 \%)$ & $6(14.6 \%)$ & \\
\hline No & $32(94.1 \%)$ & $35(85.4 \%)$ & \\
\hline
\end{tabular}

FIGO: Fédération Internationale de Gynécologie et d’Obstétrique; LVSI: lymph vascular space invasion; VSI: vascular space invasion.

Statistical analysis. All statistical analyses were performed using SPSS software (IBM SPSS Statistics, v. 25). For comparison of continuous variables such as age and tumor size among groups, Student's $t$-test was applied; ordinal variables such as FIGO stage were compared using the Mann-Whitney $U$-test, and categorical variables were compared using the chi-square test. A survival analysis was performed through the Kaplan-Meier method, and groups were compared using the log-rank test. Statistical significance was set at $p<0.05$.

\section{Results}

In total, 75 patients were included in this retrospective analysis, of whom 34 patients underwent minimally invasive surgery and 41 underwent open surgery. The mean age was 41 years in the minimally invasive group and 46 years in the open surgery group. There was no significant difference in the age of patients between the two groups, with older patients being more likely to undergo laparotomy $(p=0.057)$ (Table I). Furthermore, both groups did not show a significant difference in the histological subtype $(p=0.487)$. Tumor stage was not significantly different in both groups $(p=0.134)$.
Higher grading and higher grade of lymph vascular space invasion (LVSI) were significantly more frequent in the laparotomy group ( $p=0.035$ and $p=0.016$, respectively).

There was no significant difference in tumor size and VSI between the two groups ( $p=0.182$ and $p=0.394$, respectively). The mean follow-up time for both groups was 41 months. OS was $94.1 \%$ in the minimally invasive surgery group, and $85.4 \%$ of patients undergoing laparotomy were still alive. OS did not show a significant difference between both groups $(p=0.311)$ (Figure 1).

The mean follow-up time for disease-free survival was 39 months, with a disease-free survival (DFS) rate of $94.1 \%$ in the minimally invasive surgery group and $75.6 \%$ in the laparotomy group. There was a significant difference in favor of the laparotomy group ( $p=0.049)$ (Figure 2).

\section{Discussion}

In this hospital-based retrospective analysis, patients who underwent minimally invasive surgery showed improved 


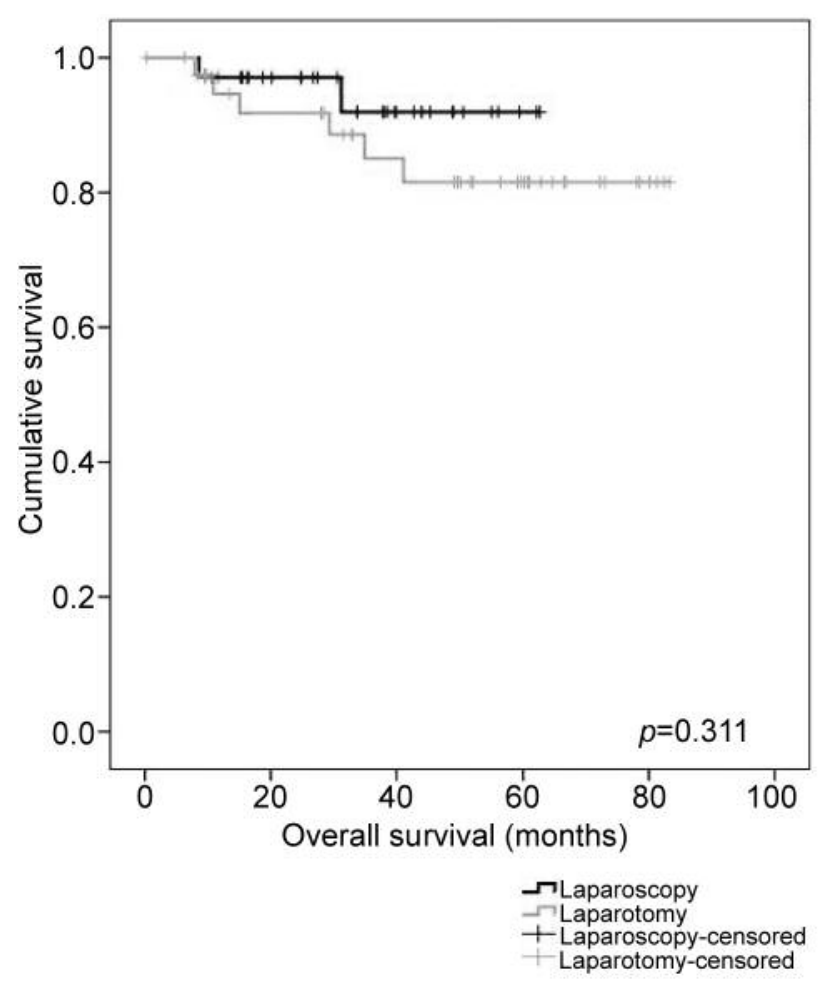

Figure 1. Overall survival in months.

DFS compared with patients who underwent open surgery (94.1\% vs. $75.6 \% ; p=0.049)$. OS was not statistically different between the groups ( $p=0.311$ ), although the OS rate was $94.1 \%$ versus $85.4 \%$ within the follow-up time of 41 months. The results presented are in contrast with previous data of LACC analysis. Ramirez and colleagues showed that minimally invasive surgery is associated with a lower rate of OS than open surgery (3-year rate, $93.8 \%$ vs. 99.0\%; $\mathrm{HR}=6.00 ; 95 \% \mathrm{CI}=1.77-20.30)$ (4). The different results can be explained by the difference in higher grading and higher grade of lymphatic infiltration between the groups, which could have influenced the results. Furthermore, the current analysis included patients with FIGO stage IIB, whereas most patients (91.9\%) in the study conducted by Ramirez et $a l$. had stage IB1 disease (4). Another main difference is that the current analysis also included patients treated with neoadjuvant therapy prior to surgery. Additionally, we did not use any type of uterine manipulator during surgery.

Wang et al. evaluated 12 studies comparing laparoscopic radical hysterectomy (754 patients) with open radical hysterectomy (785 patients) for cervical cancer. They showed no significant differences in the 5-year rate of OS $(\mathrm{HR}=0.91 ; 95 \% \mathrm{CI}=0.48-1.71 ; p=0.76)$ or the 5 -year rate of disease-free survival $(\mathrm{HR}=0.97 ; 95 \% \mathrm{CI}=0.56-1.68 ; p=0.91)$ between the two groups $(8,9)$.

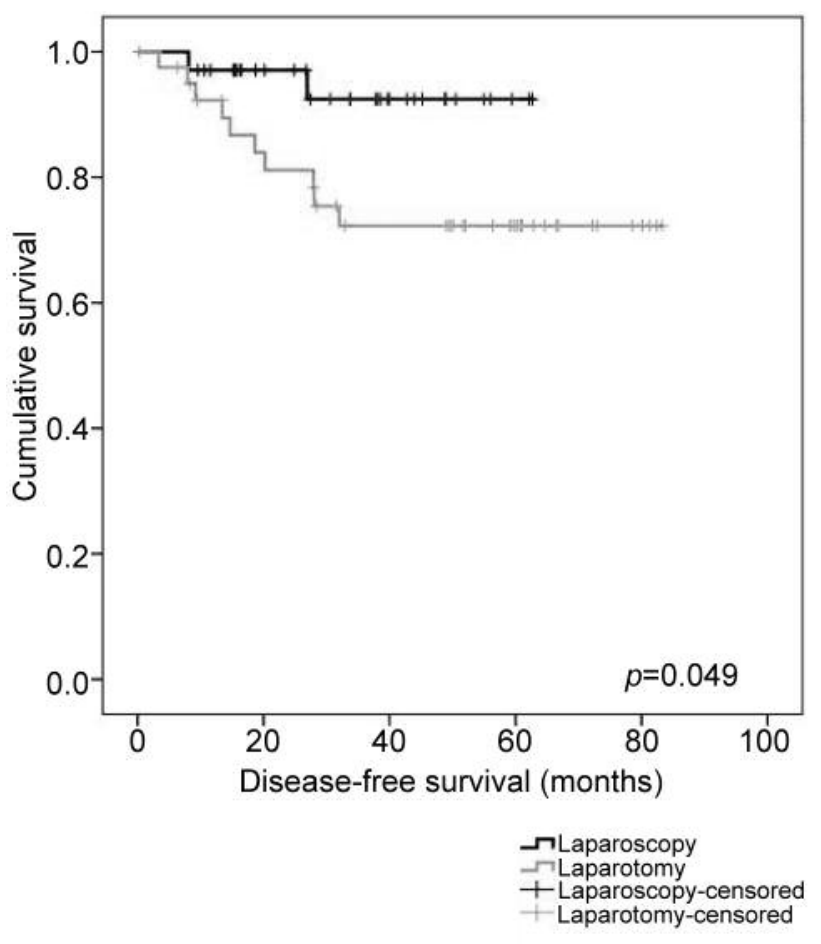

Figure 2. Disease-free survival in months.

In a meta-analysis including 22 studies involving 2,922 patients $(1,230$ underwent laparoscopic surgery and 1,692 underwent open surgery), Cao et al. showed that the diseasefree survival rate, the OS rate, and the recurrence rate did not differ significantly between the two groups $(8,10)$. These results are in concordance with those of the current analysis. Studies comparing both surgical methods as a treatment method of cervical cancer seem to be inconclusive.

Strengths and limitations. The retrospective nature is a limitation of the study, although the current analysis presented data of adjuvant and neoadjuvant treatment.

\section{Conclusion}

The results showed that minimally invasive surgical therapy for cervical cancer is not inferior to abdominal open surgery in terms of OS. Our minimally invasive surgery cohort showed improved DFS compared with the open surgery cohort. Additional prospective trials with larger cohorts are needed to confirm the results. Nevertheless, patients with FIGO stage IA1 (with LVSI), IA2, or IB1 cervical cancer have to be informed about the results of the previously published LACC study prior to making a decision on the route for radical hysterectomy in accordance with the official 
statement of the Uterus Commission of the Gynecological Oncology Working Group (AGO) (11).

\section{Conflicts of Interests}

The Authors have no conflicts of interest to declare.

\section{Authors' Contributions}

Manuscript writing: DR, CL and FT; Manuscript editing: BM, CE, BG, SL and PM; Statistical analysis: DR, CL and FT; Data collection: DR, CL, BM, CE, BG, SL, PM and FT; Project development: DR, CL, PM and FT.

\section{Acknowledgements}

The Authors thank Mrs. Kohlhagen, Mrs. Hess and Ms. Schmidt and Mrs. Schmitz for their secretarial support.

\section{References}

1 Kirn V, Zaharieva I, Heublein S, Thangarajah F, Friese K, Mayr D and Jeschke U: ESR1 promoter methylation in squamous cell cervical cancer. Anticancer Res 34: 723-727, 2014. PMID: 24511005 .

2 Lynge E, Antilla A, Arbyn M, Segnan N and Ronco G: What's next? Perspectives and future needs of cervical screening in Europe in the era of molecular testing and vaccination. Eur $\mathbf{J}$ Cancer Oxf Engl 1990 45: 2714-2721, 2009. PMID: 19695870. DOI: 10.1016/j.ejca.2009.07.024

3 Bergauer F, Brüning A, Shabani N, Blankenstein T, Jückstock J, Dian D and Mylonas I: Inhibin/activin-betaE subunit in normal and malignant human cervical tissue and cervical cancer cell lines. J Mol Histol 40: 353-359, 2009. PMID: 20033758. DOI: $10.1007 / \mathrm{s} 10735-009-9246-\mathrm{x}$

4 Liao C-J, Wu T-I, Huang Y-H, Chang T-C, Wang C-S, Tsai M-M, Hsu C-Y, Tsai M-H, Lai C-H and Lin K-H: Overexpression of gelsolin in human cervical carcinoma and its clinicopathological significance. Gynecol Oncol 120: 135-144, 2011. PMID: 21035170. DOI: 10.1016/j.ygyno.2010.10.005

5 Kim J, Kim BK, Lee CH, Seo SS, Park S-Y and Roh J-W: Human papillomavirus genotypes and cofactors causing cervical intraepithelial neoplasia and cervical cancer in Korean women. Int J Gynecol Cancer Off J Int Gynecol Cancer Soc 22: 1570-1576, 2012. PMID: 23051954. DOI: 10.1097/IGC.0b 013e 31826aa5f9
6 Leitlinienprogramm Onkologie: Zervixkarzinom. Available from: https://www.leitlinienprogramm-onkologie.de/leitlinien/ zervixkarzinom/ [last accessed February 5, 2019].

7 Tewari KS, Sill MW, Long HJ, Penson RT, Huang H, Ramondetta LM, Landrum LM, Oaknin A, Reid TJ, Leitao MM, Michael HE and Monk BJ: Improved survival with bevacizumab in advanced cervical cancer. N Engl J Med 370: 734-743, 2014. PMID: 24552320. DOI: 10.1056/NEJMoa1309748

8 Ramirez PT, Frumovitz M, Pareja R, Lopez A, Vieira M, Ribeiro R, Buda A, Yan X, Shuzhong Y, Chetty N, Isla D, Tamura M, Zhu T, Robledo KP, Gebski V, Asher R, Behan V, Nicklin JL, Coleman RL and Obermair A: Minimally Invasive versus Abdominal Radical Hysterectomy for Cervical Cancer. N Engl J Med 379: 1895-1904, 2018. PMID: 30380365. DOI: 10.1056/ NEJMoa1806395

9 Wang Y, Deng L, Xu H, Zhang Y and Liang Z: Laparoscopy versus laparotomy for the management of early stage cervical cancer. BMC Cancer 15: 928, 2015. PMID: 26596955. DOI: 10.1186/s12885-015-1818-4

10 Cao T, Feng Y, Huang Q, Wan T and Liu J: Prognostic and Safety roles in laparoscopic versus abdominal radical hysterectomy in cervical cancer: A meta-analysis. J Laparoendosc Adv Surg Tech A 25: 990-998, 2015. PMID: 26584414. DOI: 10.1089/lap.2015.0390

11 Hillemanns P, Brucker S, Holthaus B, Kimmig R, Lampe B, Runnebaum I, Ulrich U, Wallwiener M, Fehm T, Tempfer C and AGO Uterus and the AGE of the DGGG: Updated opinion of the Uterus Commission of the Gynecological Oncology Working Group (AGO) and the Gynecological Endoscopy Working Group (AGE) of the German Society of Gynecology and Obstetrics (DGGG) on the randomized study comparing minimally invasive with abdominal radical hysterectomy for early-stage cervical cancer (LACC). Geburtshilfe Frauenheilkd 79: 145-147, 2019. PMID: 30792544. DOI: 10.1055/a-0824-7929
Received April 1, 2019

Revised April 17, 2019

Accepted April 22, 2019 